\title{
Der Zusammenhang von Kieferorthopädie und Sprechauffälligkeiten
}

\section{The Relationship Between Orthodontics and Speech Anomalies}

\author{
Autoren \\ Sandra Riemekasten', Birke Peter², Michael Fuchs ${ }^{3}$ \\ Institute \\ 1 Poliklinik für Kieferorthopädie, Universität Leipzig \\ 2 Sektion für Phoniatrie und Audiologie / Praxis für \\ Stimm- und Sprachtherapie, Universitätsklinikum Leipzig \\ 3 Sektion Phoniatrie und Audiologie, Cochlea-Implantat- \\ Zentrum, Universitätsklinikum Leipzig \\ Schlüsselwörter \\ orofaziale Störungen, myofunktionelle Störungen, \\ Sprachentwicklung \\ Key words \\ orofacial disorders, myofunctional disorders, \\ speech development \\ Bibliografie \\ DOI https://doi.org/10.1055/a-0830-1821 \\ Inf Orthod Kieferorthop 2019; 51: 51-63 \\ (c) Georg Thieme Verlag KG Stuttgart · New York \\ ISSN 0020-0336
}

Korrespondenzadresse

Dr. med. dent. Sandra Riemekasten

Poliklinik für Kieferorthopädie

Universität Leipzig

Liebigstraße 12, Haus 1

04103 Leipzig

Sandra.Riemekasten@medizin.uni-leipzig.de

\section{ZUSAMMENFASSUNG}

Erkenntnisse aus der Kontrolle der Gebissentwicklung sind elementar für Ursachenfindung und Therapie von Zahn- und Kieferfehlstellungen, können jedoch ebenso durch diese bedingt sein. Dieser Artikel soll Grundlagen für die Diagnostik und Therapie solcher Störungen darlegen, das Grundwissen hierzu erweitern und eventuelle Wissenslücken auffüllen.

\section{ABSTRACT}

Findings from the control of jaw development are elementary for detecting the cause and therapy of tooth misalignment and malpositions of the jaw, but they can also be provoked by them. This article is intended to provide basic knowledge or fill in any gaps for the diagnosis and treatment of such disorders.

\section{Einführung}

Eine regelmäßige Kontrolle der Gebissentwicklung in den Phasen des Milch- und Wechselgebisses sowie der rechtzeitige Beginn einer erforderlichen kieferorthopädischen Therapie sollten bei jedem Kind routinemäßig vorgesehen werden. Nicht selten werden im Zuge dessen zusätzliche Störungen im orofazialen Bereich festgestellt. Besonderes Augenmerk ist dabei auf myofunktionelle Störungen, die Sprachentwicklung, die Stimme sowie auf Haltung und das Schlucken zu legen.

\section{Normgerechte Entwicklung der Kiefer - Übersicht physiologische Entwicklung des Gebisses}

Für die Erkennung nicht normgerechter, also „dysgnather“ Zahnund Kieferfehlstellungen muss man sich zunächst der normalen Entwicklung dieser Bereiche widmen:
Bei der Geburt eines Kindes befindet sich der Unterkiefer regelmäßig meist noch in einer dezenten Rücklage, die während des Weiteren Wachstums ausgeglichen wird [1]. Zu diesem Zeitpunkt haben die Babys üblicherweise noch keine Zähne im Mund. Um die „normgerechte“ Entwicklung des Gebisses der Menschen einschätzen zu können, wurden verschiedene Schemata erstellt [2-4], die eine entsprechende Übersicht über den regelgerechten Durchbruch der Zähne bieten.

Üblicherweise sind es die mittleren Schneidezähne, die im Alter von ca. 6-8 Monaten in die Mundhöhle durchbrechen, wobei die Zahnung meist im Unterkiefer beginnt. Es folgen die seitlichen Milchschneidezähne, Milcheckzähne und Milchmolaren, sodass ein vollständiges Milchgebiss im Alter von ca. 30 Monaten vorliegt.

Ab einem Alter von ca. 6 Jahren beginnt der Zahnwechsel, zunächst in der sogenannten 1. Phase des Wechselgebisses, mit dem Durchbruch der permanenten Frontzähne und der 6-Jahres-Molaren, welche hinter der Milchzahnreihe durchdringen. 
- Tab. 1 Zahndurchbruch nach Alter.

\begin{tabular}{|l|l|}
\hline Alter in Jahren & Zähne im Wechsel \\
\hline $6-7$ & $\begin{array}{l}\text { 1. Molaren OK/UK mittlere Schneidezähne UK } \\
\text { mittlere Schneidezähne OK seitliche Schneidezäh- } \\
\text { ne OK/UK }\end{array}$ \\
\hline $7-9$ & $\begin{array}{l}\text { Eckzähne und Prämolaren } \\
\text { 2. Molaren OK/UK }\end{array}$ \\
\hline $10-12$ & 3. Molaren OK/UK \\
\hline $12-13$
\end{tabular}

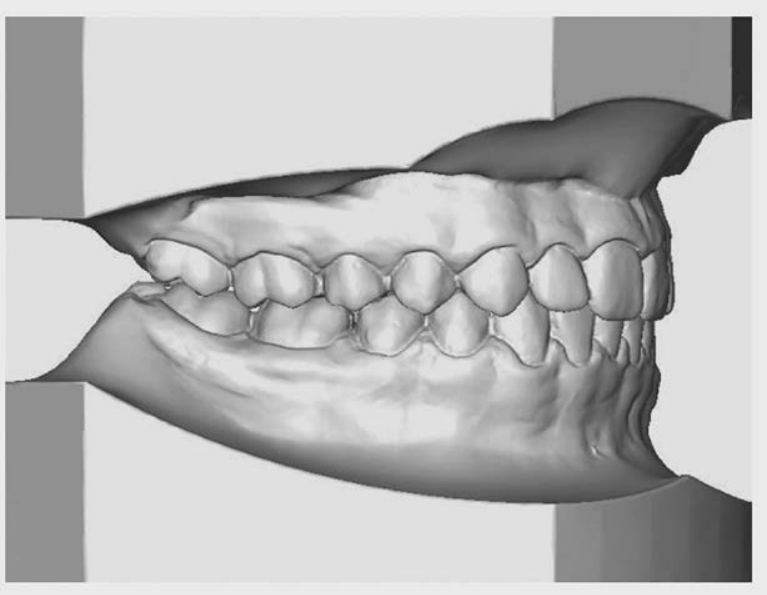

Abb. 1 Eugnathes Gebiss.

- Tab. 2 Sprachsystematische Ebenen.

\begin{tabular}{|l|l|}
\hline Ebene & \\
\hline $\begin{array}{l}\text { phonetisch- } \\
\text { phonologisch }\end{array}$ & $\begin{array}{l}\text { phonetisch: Lautbildung, Laute können motorisch } \\
\text { gebildet werden phonologisch: Lautverwendung, } \\
\text { Laute können korrekt in ihre Lautumgebung } \\
\text { eingesetzt werden }\end{array}$ \\
\hline $\begin{array}{l}\text { semantisch- } \\
\text { lexikalisch }\end{array}$ & $\begin{array}{l}\text { Begriffsbildung/Sprachverständnis aktiver und } \\
\text { passiver Wortschatz }\end{array}$ \\
\hline $\begin{array}{l}\text { morphologisch- } \\
\text { syntaktisch }\end{array}$ & $\begin{array}{l}\text { Verständnis und Gebrauch grammatikalischer } \\
\text { Regeln }\end{array}$ \\
\hline $\begin{array}{l}\text { pragmatisch- } \\
\text { kommunikativ }\end{array}$ & $\begin{array}{l}\text { Fähigkeit zu einer situationsadäquanten } \\
\text { Kommunikation Organisation von Kommunikation }\end{array}$ \\
\hline
\end{tabular}

Die 2. Phase des Wechselgebisses, welche durch den Durchbruch des bleibenden Seitenzahngebiets gekennzeichnet ist, beginnt mit ca. 10 Jahren und ist mit ca. 12-14 Jahren beendet, bei Mädchen im Durchschnitt ca. 2 oder 3 Jahre eher als bei Jungen ( $>$ Tab. 1 ).

Nach Abschluss der Zahnung und des Wachstums sollte ein eugnathes Gebiss vorliegen. Der Begriff „eugnath“ stammt aus dem Altgriechischen und bedeutet so viel wie ein „guter“ Kiefer.
Doch was heißt in diesem Fall „gut"? Gekennzeichnet ist ein eugnathes Gebiss durch verschiedene Merkmale [4, 5]. Auf den gut ausgeformten Zahnbögen sollte die vollständige Zahnzahl vorhanden sein, wobei die Zähne selbst eine normgerechte Zahnform aufweisen, keine Engstände oder Lücken oder andere (Einzel-)Zahnabweichungen vorliegen und sich die Zähne in korrekter vertikaler Position befinden. Die Kieferbasen sollten eine angemessene Größe haben und korrekt in den Schädel einlagert sein. Die Kiefer sollten in neutraler Position zueinander stehen, d. h. die Spitze des oberen Eckzahns sollte zwischen den unteren Dreier und Vierer weisen, der mesiale Höcker des oberen 1. Molaren sollte in die bukkale Querfissur des unteren 1. Molaren zeigen ( $\triangleright$ Abb. 1). Die Frontzähne sollten einen Abstand von $2 \mathrm{~mm}$ in der Vertikalen und Sagittalen aufweisen.

Zu beachten ist, dass nur 2-3\% der Population tatsächlich über ein wirklich eugnathes Gebiss verfügen. Sollten Abweichungen von diesem idealen Gebiss und der Artikulation z. B. durch den Zahnarzt festgestellt werden, wird eine Überweisung zum Fachzahnarzt für Kieferorthopädie empfohlen. Dieser wird entscheiden, welche Problematik bei der Abweichung vom eugnathen Gebiss vorliegt und ob eine kieferorthopädische Behandlung des Patienten notwendig ist.

Ein eugnathes Gebiss ist gekennzeichnet durch [1]:

- gut ausgeformte Zahnbögen

- vollständige Zahnzahl

- normgerechte Zahnform

- keine Engstände oder Lücken

- keine Einzelzahnabweichungen

- Zähne in korrekter vertikaler Position

- Kieferbasen mit angemessener Größe

- korrekte Einlagerung der Kieferbasen in den Schädel

- Kiefer in neutraler Position

- Frontzähne im Abstand von 2 mmin der Vertikalen und Sagittalen

\section{Übersicht physiologische Sprachentwicklung - Fokus phonetischphonologische Ebene}

Fehlstellungen der Zähne und des Kiefers können mit Beeinträchtigungen der Sprachentwicklung, im Besonderen der Artikulation, der Lautbildung und myofunktionellen Störungen assoziiert sein. Um entscheiden zu können, welche Art der Beeinträchtigung vorliegt, sind Kenntnisse über Grundzüge der kindlichen Sprachentwicklung wertvoll. Daher sollen im Folgenden Meilensteine der kindlichen Sprachentwicklung dargestellt werden.

Bei der Untersuchung der Sprachentwicklung bei Kindern werden die sprachsystematischen Ebenen beurteilt ( $\vee$ Tab. 2). Hierbei handelt es sich um die phonetischphonologische, die semantischlexikalische, die morphologisch-syntaktische und pragmatischkommunikative Ebene. 
Aus Gründen einer übersichtlicheren Darstellung werden keine Ausführungen zur pragmatisch-kommunikativen Ebene vorgenommen.

\section{Meilensteine der Sprachentwicklung}

Vorsprachliche Fähigkeiten, 1. und 2. Lallphase

\section{Lallphase bis ca. 6./8. Lebensmonat}

Diese Phase ist gekennzeichnet durch Schreien und Gurren, später dann auch durch Lachen. Im weiteren Verlauf werden Gurgel-, Schmatz- und Vokallaute produziert. Bei der Bildung dieser lautlichen Äußerungen stehen die Tastinformationen der Artikulationsorgane im Vordergrund, die motorische Geschicklichkeit wird trainiert. Das Funktionsspiel der Stimme setzt ein. Damit ist z. B. eine variierte ununterbrochene oder unterbrochene Stimmgebung mit Variation in Dauer, Intonation und Lautstärke gemeint. In dieser Phase spielt die eigene Kontrolle über das Gehör noch keine wesentliche Rolle. Auch hörgeschädigte Kinder durchlaufen die 1. Lallphase.

\section{Lallphase 6./8. bis ca. 12. Lebensmonat}

Diese Phase ist vom Hören gesteuert. Die Bildung der Laute und Prosodie/Betonungsmuster nähert sich immer mehr der Muttersprache an. Die Aufmerksamkeit wird auf die eigene Lautproduktion gelenkt, da dies nun durch akustische Kontrolle ermöglicht wird. Bei hörgeschädigten Kindern ist eine Reduktion der Annäherung der Lautbildung und Prosodie an die Muttersprache bzw. sogar ein Verstummen zu verzeichnen.

Im Bereich der Sprachproduktion sind Lallmonologe zu verzeichnen. Die Produktion von muttersprachlichen Silben und Silbenketten („dada“, „baba“), Imitation von Lautkombinationen und muttersprachliche Prosodie/Betonungsmuster sind Inhalte der 2. Lallphase.

Nachahmung von Geräuschen und die Produktion erster Wörter, wie z. B. „Mama“, „nein“, „wau-wau“, können beobachtet werden. Auf der Ebene des Sprachverständnisses reagiert das Kind auf seinen Namen und auf einfache Aufforderung, wie z. B. „Komm her!".

Der Einsatz von Gesten entwickelt sich bzw. nimmt zu. Die Nachahmung von Gesten wie z. B. „winke-winke“ sind zu beobachten. Es wird auf Gegenstände gezeigt, die das Kind haben möchte. Kopfschütteln, wenn es etwas nicht will; Nicken, wenn es einverstanden ist.

\section{Bis zum 2. Lebensjahr}

\section{Phonetisch-phonologische Ebene}

Auf dieser Ebene erfolgt die Bildung bilabialer Laute, z. B. /m/, /b/, /d/, /t/, /n/, /p/, /f/, /w/, /l/.

\section{Lexikalisch-semantische Ebene}

Es vollzieht sich der Beginn des Wortschatzaufbaus, erste Wörter wie z. B. „Mama, Papa, Ball, Auto“ können gesprochen werden. Um den 24. Monat besteht ein aktiver Wortschatz von 50 Wörtern. Kinder, die diese Marke unterschreiten, sind sogenannte Late Talker. Um dies zu überprüfen, werden durch Sprachtherapeuten validierte Elternfragebögen eingesetzt. Üblicherweise findet bis hierher jedoch eine sogenannte Wortschatzexplosion statt, außerdem werden einfache Aufforderungen z. B. „Gib mir den Ball“, „Wo ist der Hund/Wauwau?“ nunmehr verstanden. Erfasst werden ca. 100200 Wörter.

\section{Morphologisch-syntaktische Ebene}

Das Kind nutzt Einwortsätze, d. h. es kann mit einem Wort feststellen, erbitten, fragen, antworten. Beispiele hierfür sind z. B. „Auto“ für „Da ist ein Auto zu sehen“, „Auto!“ für „Ich möchte mit dem Auto da spielen!“, „Auto?“ für „Habe ich da ein Auto gehört?“. Zu Beginn des 2. Lebensjahres werden Zwei- und vereinzelt auch Dreiwortsätze, z. B. „Auto weg“, „Mama da“, gebildet.

\section{Bis zum Alter von 3 Jahren}

\section{Phonetisch-phonologische Ebene}

Es erfolgen erste Konsonantenverbindungen (z. B. /bl/, / fl/). Lexikalisch-semantische Ebene. Das Kind spricht mit 30 Monaten etwa 450 Wörter, unter Nutzung erster Präpositionen (z. B. „auf“, „unter“), Personalpronomen (z. B. „ich“, „du“, „mein“). Es versteht außerdem Zweifachaufträge, z. B. „Leg das Auto auf den Tisch“. Die Zuordnung von Grundfarben ist möglich.

\section{Morphologisch-syntaktische Ebene}

Eine korrekte Verbzweitstellung, z. B. in „Papa isst Eis“ ist nun ebenso möglich wie Nebensatzbildungen mit einfachen Konjunktionen (z. B. „und“, „weil“). Hier findet man auch das zweite Fragealter („Wer? Was? Wo? Warum?“) und es werden Drei- bis Fünfwortsätze gebildet.

\section{Bis zum Alter von 4 Jahren}

\section{Phonetisch-phonologische Ebene}

Mit vollendetem 4. Lebensjahr sollten alle Laute bis auf das /s/ und / sch/korrekt ausgesprochen werden.

\section{Lexikalisch-semantische Ebene}

Die Nutzung und das Verständnis von Präpositionen (z. B. „neben“, „vor“) nimmt zu, Farben werden korrekt benannt und Mehrfachaufträge, z. B. „ Hol den roten Ball und gib ihn mir“, verstanden.

\section{Morphologisch-syntaktische Ebene}

Auffällig ist hier die korrekte Verbzweit-/endstellung in Hauptund Nebensätzen (z. B. „Ich möchte trinken, weil ich Durst habe“.). Die Verbflexion ist überwiegend korrekt (z. B. „Ich mache ...“, „Mama macht ...“). Es werden Vergangenheits- und Zukunftsformen verwendet sowie Lieder gesungen und kleine Verse und Reime gesprochen.

\section{Bis zum Alter von 5 Jahren}

\section{Phonetisch-phonologische Ebene}

Alle Laute werden korrekt gesprochen, außer dem S-Laut.

\section{Lexikalisch-semantische Ebene}

Hier werden Oberbegriffe wie z. B. Obst, Möbel und abstrakte Begriffe, wie z. B. Glück, verwendet. Das Kind kann seinen Vor- und Nachnamen nennen, bis 10 zählen und Aufträge in korrekter Reihenfolge, z. B. „Nimm die große Puppe und setze sie auf den roten Stuhl“, erfüllen.

\section{Morphologisch-syntaktische Ebene}

Es findet die korrekte Verwendung von Pluralformen/ohne Stammvokalveränderung, z. B. „der Finger/die Finger“, statt. Folgendes ist zu beachten: der korrekte Gebrauch von Formen, die im Stammvokal (z. B. „der Zahn/die Zähne“) verändert sind, kann sich bis ins 10. Lebensjahr hinziehen. Bei verneinenden Sätzen sowie Frage- und Passivsätzen zeigen sich noch Unsicherheiten. Kleine Geschichten können nacherzählt werden. 


\section{Bis zum 6. Lebensjahr}

\section{Phonetisch-phonologische Ebene}

Die Bildung und der Einsatz aller Laute sind normgerecht, mit Ausnahme der S-Laute.

\section{Lexikalisch-semantische Ebene}

Es findet eine weitere Ausdifferenzierung des Wortschatzes statt, die Verwendung abstrakter Begriffe ist nun sicher möglich. Morphologisch-syntaktische Ebene

Muttersprachliche grammatikalische Regeln werden beherrscht. Die Übermittlung von Gedankengängen ist kindgerecht möglich. Die Wiedergabe von Geschichten und Erlebnissen erfolgt zusammenhängend, auch unter Nutzung verschiedener Zeitformen.

\section{Ursachen und Symptome von Sprachentwicklungsstörung}

Eine Sprachentwicklungsstörung (SES) liegt vor, wenn es auf einer oder mehreren sprachsystematischen Ebenen signifikante zeitliche und inhaltliche Abweichungen von der Altersnorm nach unten gibt. Die Beeinträchtigung der Sprachentwicklung kann isoliert oder im Zusammenhang mit weiteren Störungen bzw. Primärerkrankungen auftreten und führt zur Beeinträchtigung der Entwicklung des Kinds.

Bei weiteren Störungen bzw. Primärerkrankungen können folgende Grunderkrankungen von Bedeutung sein, z. B.:

- Intelligenzminderung

- Hörstörung

- andere Sinnesbehinderungen und bei Mehrfachbehinderungen

- tief greifenden Entwicklungsstörungen, z. B. Autismus

- in Vergesellschaftung mit Syndromen, z. B. Trisomie 21

- Störungen der motorischen Funktionen

Die Abklärung der Sprachentwicklung kann durch einen Sprachtherapeuten erfolgen. Weiterhin sollte, wenn der Verdacht einer nicht altersgerechten Sprachentwicklung im Raum steht, grundsätzlich eine fachärztliche Untersuchung durch einen Phoniater und Pädaudiologen oder durch einen HNO-Arzt erfolgen, insbesondere zum Ausschluss einer Hörstörung, einer larvierten/submukösen Gaumenspalte und zur Beurteilung der adenoiden Vegetation.

\section{Warum ist diese Abklärung dringend anzuraten? \\ Hörminderung}

Eine Hörminderung kann vor allem zu Auffälligkeiten auf phonetisch-phonologischer Ebene führen, da keine ausreichende auditive Rückkopplung, also akustische Kontrolle der Artikulation, möglich ist. Bei einer Hörstörung im Hochtonbereich sind insbesondere oft die sogenannten Sibilanten, d. h. hochfrequente Laute wie /s/ Laute, /sch/, / $\mathrm{ch}^{1} /$ ( $\mathrm{ch}^{1}$ meint die Bildung des /ch/ wie in ich, $\mathrm{ch}^{2}$, meint die Bildung des /ch/ wie in auch), betroffen. Bei fehlender akustischer Rückkopplung werden diese Laute oft undeutlich, verwaschen artikuliert oder gar gänzlich getilgt. Auch stimmlose Konsonanten, vor allem in wortfinaler Stellung, sind oft davon betroffen, z. B. „Hau“ statt „Haus“, „Hun“ statt „Hund“.

Submuköse Gaumenspalte (SMGS)

Beim Vorliegen einer SMGS kann es auch auf phonetischphonologischer Ebene zu einer nicht altersgerechten Entwicklung kommen.
Die Bildung der velaren Laute $(/ \mathrm{g} /, / \mathrm{k} /, / \mathrm{ng} /, / \mathrm{r} /)$ kann beeinträchtigt sein.

Offenes Näseln (Rhinolalie/Rhinophonia aperta) ist aufgrund der velopharyngealen Insuffizienz und der reduzierten Gaumenkontraktion als Leitsymptom möglich. Auch eine Tubenfunktionsstörung, die durch die eingeschränkte Belüftung zu chronischen Veränderungen im Mittelohr und zu Höreinschränkungen führen kann, ist ein typisches Symptom.

\section{Adenoide Vegetation}

Die adenoide Vegetation (syn. hypertrophe Rachenmandel) kann zu einer Behinderung der Nasenatmung führen. Kinder, die davon betroffen sind, entwickeln häufig eine myofunktionelle Störung. Konsekutiv kann es dabei auch zu einer Zahnfehlstellung, z. B. einem sogenannten „offenen Biss“, kommen.

Die adenoide Vegetation kann über den Pathomechanismus der Minderbelüftung der Tuba auditiva und des Mittelohres zu einem Tubenmittelohrkatarrh mit Paukenerguss führen, woraus sich nachfolgend eine Schallleitungsschwerhörigkeit entwickeln kann.

Bei weiteren Fragestellungen bezüglich der Sprachentwicklung oder Persistenz der Sprachentwicklung ist weiterführend eine kognitive, psychologische und motorische Abklärung anzuraten.

Im Folgenden sollen einige Hinweise für das Erkennen einer anamnestisch verzögerten Sprachentwicklung benannt werden:

- später Sprachbeginn (Es wird berichtet, das Kind hat erst mit 2 oder 21ํㄹ Jahren angefangen, erste Worte zu sprechen.)

- langsamer Spracherwerb mit möglicher Plateaubildung, Sprachverständnis besser als Sprachproduktion

- andere Komorbiditäten, die als Grunderkrankung eine verzögerte Sprachentwicklung erklären können

Die Ursachen für eine SES können sehr unterschiedlich sein und nicht immer ist es möglich, diese eindeutig zu ermitteln. Sie treten häufig und oftmals kombiniert auf. Ursachen dafür können unter anderem sein:

- häufig wiederkehrende Mittelohrentzündungen im frühen Kindesalter

- Beeinträchtigungen des Hörvermögens

- allgemeine Entwicklungsverzögerung/-störung

- Fehlbildungen im Gesichtsbereich (z. B. Lippen-, Kiefer-, Gaumenspalten)

- mangelndes Sprachangebot im sozialen Umfeld

- genetische Faktoren

- Intelligenzminderung

- frühkindliche Hirnschädigungen

- psychische Beeinträchtigungen wie z. B. ADHS, Autismus

Wenn sprachliche oder artikulatorische Beeinträchtigungen festgestellt werden, die einen Bezug zur Kiefer- und Zahnstellung haben können, sollten unbedingt folgende Krankheitsbilder fachärztlich (Phoniatrie und Pädaudiologie oder HNO-Heilkunde) abgeklärt werden:

- Hörstörung

- adenoide Vegetation

- submuköse Gaumenspalte 


\section{Fokus Diagnostik - Aufgaben eines Kieferorthopäden und Zahnarztes}

Zahnärzte und Kieferorthopäden beschäftigen sich auch mit der Überwachung der Zahn- und Kieferentwicklung von Kindern und Jugendlichen. Im Zuge der Diagnostik erfolgt die Bestimmung des optimalen Behandlungsbeginnes sowie der kieferorthopädischen Behandlung und Retention des kieferorthopädischen Behandlungsergebnisses im Wechsel- und permanenten Gebiss. Die Therapie kann sowohl mit herausnehmbaren als auch mit festsitzenden Apparaturen durchgeführt werden.

Zunächst erfolgt eine umfassende Anamnese des Patienten. Dabei sollte der Patient nicht nur intraoral, sondern ganzheitlich betrachtet werden. Dies ist ein grundlegender Aspekt eines jeden Arztbesuches. Schauen Sie auf die Haltung des Patienten, seinen Tonus. Vielleicht erkennen Sie sogar orthopädische Auffälligkeiten beim Gehen, Stehen oder Sitzen. Es ist also enorm wichtig, den Blick auch einmal fernab der Zähne schweifen zu lassen und den gesamten Körperbau, die gesamte Haltung des Patienten zu betrachten.

Schließlich ist es für den Kieferorthopäden unabdingbar, den Patienten intraoral zu begutachten und dessen Behandlungsnotwendigkeit einzuschätzen. Mit Vorstellung des Patienten beim Kieferorthopäden kann festgestellt werden, in welche Stufe der kieferorthopädischen Indikationsgruppen (KIG) die Fehlstellung und der Schweregrad der Fehlstellung des Patienten einzuordnen ist [6].

Seit dem 1. Januar 2002 gelten die vorgenannten kieferorthopädischen Indikationsgruppen als Richtlinie, aufgrund derer die Entscheidung fällt, ob die Behandlung medizinisch notwendig und der Schweregrad der Anomalie erreicht ist, sodass die daraus anfallenden Kosten durch die Krankenkasse übernommen werden oder ob diese anderenfalls privat im Rahmen der Gebührenordnung für Zahnärzte zu zahlen sind. Diese Regelung findet bis zum 18. Lebensjahr Anwendung.

Die Hauptbehandlungsphase für Kieferorthopäden ist die 2. Phase des Wechselgebisses. Hier brechen die permanenten Zähne durch und das Wachstum ist noch nicht abgeschlossen. Dabei gibt es 11 verschiedene Gründe, die ab Schweregrad 3 zu einem von der Krankenkasse bezuschussten Behandlungsbedarf führen. Durch die Krankenkassen werden die Kosten der Behandlung bei folgenden Diagnosen übernommen [6]:

- kraniofazialen Anomalien

- Zahnunterzahlen

- Durchbruchsstörungen (Retention, Verlagerung)

- vergrößerten sagittalen Stufen (>6 mm)

- progener Verzahnung

- offenem Biss (>2 mm)

- tiefem Biss (>3 mm mit traumatischem Gingivakontakt)

- transversalen Einzelzahnabweichungen (Bukkal-, Lingualokklusion)

- Kreuzbissen

- sowie bestimmten Graden an Engständen und Platzmangel für die durchbrechenden Zähne

Darüber hinaus gibt es Ausnahmefälle, in denen eine Behandlung schon vor dem Zeitpunkt der 2. Phase des Wechselgebisses vorgenommen werden kann. Diese betreffen vergrößerte sagittale Stufen über $9 \mathrm{~mm}$, progene Verzahnungen und skelettal offene Bisse.
Im Rahmen einer gründlichen Untersuchung des Patienten kann eine Einstufung in das KIG-System durch den Facharzt für Kieferorthopädie erfolgen und eine Behandlung nach Einreichung eines Behandlungsplanes sowie Genehmigung durch die Krankenkasse begonnen werden. Die Möglichkeit, eine kieferorthopädische Behandlung als Privatzahler wahrzunehmen (v.a. ästhetische Gründe), besteht grundsätzlich immer.

Da der Kieferorthopäde ebenso Auffälligkeiten im Rahmen der Myofunktion oder Artikulation erkennt, sollte eine begleitende logopädische Behandlung bzw. eine Überweisung zum Sprachtherapeuten zur genaueren Diagnostik erfolgen. Sofern noch nicht durchgeführt und wenn sich anamnestisch eine Relevanz zeigt, sollte auch eine Vorstellung beim Hals-Nasen-Ohren-Arzt/Phoniater und Pädaudiologen eruiert werden.

Für eine erfolgreiche Therapie und eine möglichst geringe Rezidivrate müssen sämtliche nachteilige Einflussfaktoren abgestellt werden.

Besonders bei folgenden Auffälligkeiten sollte eine Überwachung durch den Kieferorthopäden stattfinden: kraniofaziale Anomalien, Zahnunterzahlen, Durchbruchsstörungen, vergrößerte sagittale Stufen, progene Verzahnung, offener Biss, tiefer Biss, transversale Einzelzahnabweichungen, Kreuzbisse, Engstände und Platzmangel für die durchbrechenden Zähne.

\section{Fokus Diagnostik - Aufgaben eines Stimm- und Sprachtherapeuten}

Im klinischen Alltag werden den akademischen Sprachtherapeuten oder Logopäden immer wieder Kinder zur Mitbeurteilung der Sprachentwicklung, der Artikulation und zur Abklärung einer myofunktionellen Störung von Zahnärzten- oder Kieferorthopäden zugewiesen. In der überwiegenden Zahl der Vorstellungen werden die Kinder und Jugendlichen vorgestellt, weil sie eine auffällige Lautbildung zeigen bzw. der Verdacht einer myofunktionellen Störung vorliegt.

Es sei nochmals betont, dass bei der Beurteilung der Sprachentwicklung, Artikulation oder einer myofunktionellen Störung eine vorherige Vorstellung beim HNOArzt/Phoniater und Pädaudiologen anzuraten ist, um Miterkrankungen, die sich ggf. anamnestisch gezeigt haben, abklären zu lassen. Es ist wichtig zu überprüfen, ob es sich hierbei um eine Störung der Artikulation (phonetische Störung) oder um eine Störung der Lautverwendung (phonologische Störung) handelt.

Folgende Fragen stellen sich:

- Wann sprechen wir von einer phonetischen, wann von einer phonologischen Störung?

- Warum ist es wichtig, diese Unterscheidung vorzunehmen?

- Welche therapeutischen Konsequenzen ergeben sich daraus?

Unter Artikulation versteht man die Formung der Sprachlaute im Vokaltrakt. Die Laute entstehen dabei durch die Bewegung der $\mathrm{Ar}$ tikulationsorgane zu den Artikulationsorten. Durch eine unterschiedliche Ausformung des Mundraumes und der Nasenhöhle 
werden die durch die Stimmlippen gebildeten Töne geformt und verändert. Aber was genau ist ein Vokaltrakt, was sind Artikulationsorgane und Artikulationsorte?

\section{Vokaltrakt (auch Ansatzraum, ( Abb. 2)}

Unter Vokaltrakt versteht man Resonanzräume, die nicht unmittelbar mit der Schallquelle verbunden sind. Beim Menschen ist das der oberhalb der Stimmlippen befindliche gesamte Hohlraumkomplex. Die Hohlräume sind miteinander gekoppelt und im Quer-

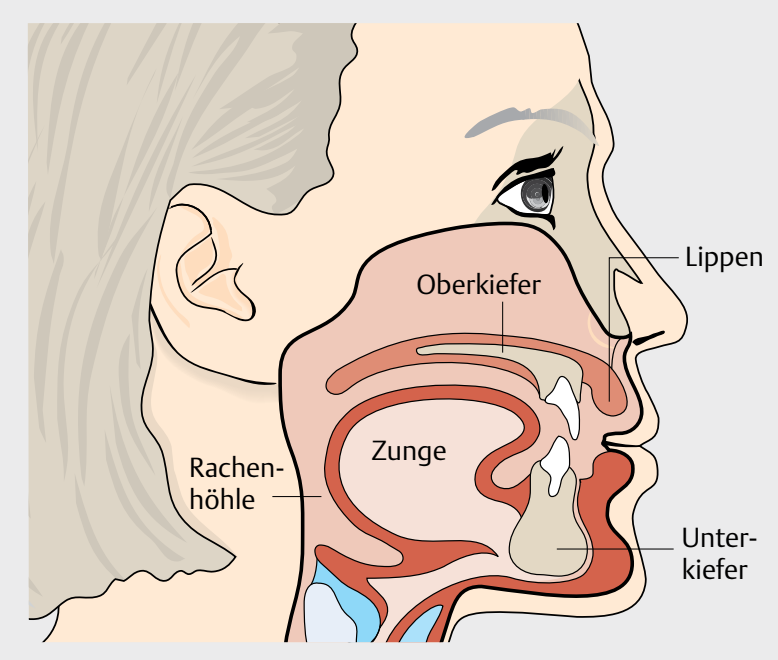

Abb. 2 Der Vokaltrakt.

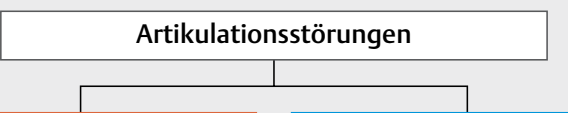

organische Ursache, z.B. audiogen bedingt kraniofaziale Anomalien

Abb. 3 Übersicht Artikulationsstörungen.

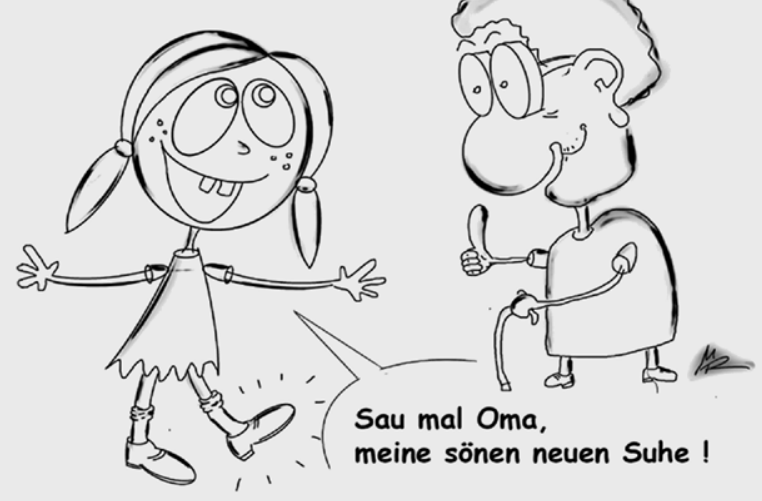

Abb. 4 Beispiel für Vorverlagerung des /sch/. schnitt jeweils veränderbar. Es handelt sich um den Naso-, Oro- und Hypopharynx, die Mundhöhle und die Nasenhaupt- und -nebenhöhlen.

\section{Artikulationsorgane}

Zu den Artikulationsorganen zählen die beweglichen Teile im Vokaltrakt:

- Lippen (Labia),

- Unterkiefer (Mandibula),

- Zunge (Lingua),

- Gaumensegel (Velum),

- Rachen (Pharynx).

\section{Artikulationsorte}

Als Artikulationsorte werden die Stellen im Ansatzraum bezeichnet, auf die sich die Artikulationsorgane zubewegen können:

- Lippen (labial),

- Zähne (dental),

- Zahndamm (alveolar),

- weicher Gaumen (velar),

- harter Gaumen (palatal),

- Gaumenzäpfchen (uvular),

- Rachen (pharyngeal),

- Kehlkopf (laryngeal).

\section{Symptome einer Artikulationsstörung (phonetische Störung)}

Von einer Artikulationsstörung wird gesprochen, wenn der motorische Vorgang der Lautbildung, die Fähigkeit, einen Laut korrekt zu bilden, betroffen ist, unabhängig davon, ob der Laut im Wort korrekt eingesetzt wird oder nicht. Grund dafür sind Defizite in der Koordination der Sprechmotorik. Die Lautbildung eines oder mehrerer Laute entspricht dabei nicht der Normphonetik der Muttersprache. Artikulationsstörungen können grundsätzlich organische oder funktionelle Ursachen haben ( $\mathbf{A b b}$. 3 ).

Bei einer phonologischen Störung ist die Lautverwendung betroffen, das heißt der Laut kann nicht korrekt in seiner Lautumgebung eingesetzt werden. Voraussetzung dafür ist, dass der Laut auch motorisch (phonetisch) gebildet werden kann. Ist dies nicht möglich, kann der Laut auch nicht in seiner Lautumgebung korrekt wiedergegeben werden und wird durch einen anderen Laut ersetzt. Aus diesem Grunde ist es für die gezielte therapeutische Behandlung wichtig zu ermitteln, ob Lautbildung oder Lautverwendung betroffen ist. Es gibt phonologische Entwicklungsprozesse, die das Kind während seiner Sprachentwicklung durchläuft und welche bis zu einem gewissen Alter überwunden werden.

Häufige physiologische Lautersetzungsprozesse im Deutschen sind [7] z. B.:

- Vorverlagerung von $/ \mathrm{g} /, / \mathrm{k} /$

- Die velaren Laute $/ \mathrm{g} /$, / $/$ / werden durch die Alveolarlaute /t/ oder/d/ ersetzt. Zum Beispiel „Kindergarten $\rightarrow$ Tindertarten“. Dieser Prozess sollte bis 3,6 Jahre, spätestens bis zum 4. Lebensjahr überwunden sein.

- Vorverlagerung von/sch/

- /sch/ wird durch ein/s/ ersetzt, oft interdental. Zum Beispiel „Schuhe $\rightarrow$ Suhe“ ( $\triangleright$ Abb. 4). Dieser Prozess sollte bis 5 Jahre, spätestens bis 5,1 Jahre überwunden sein. 


\section{Myofunktionelle Störungen (MFS)}

Myofunktionelle Störungen sind im klinischen Alltag oft mit einer Zahn- und Kieferfehlstellung vergesellschaftet. Dabei handelt es sich um eine Störung des muskulären Gleichgewichtes im Mundbereich. Davon betroffen sind Bewegungs- und Koordinationsabläufe aller am Schlucken beteiligten Strukturen aufgrund einer Fehlfunktion der Wangen-, Lippen- und Zungenmuskulatur. Sekundär können auch Störungen des Tonus und der Funktion im Ganzkörperbereich auffallen [8].

Dabei kommt es zu einer inkorrekten Zungenruhelage, zu falschem Schluckmuster und/oder Artikulationsstörungen (meist/s/, /sch/, aber auch interdentale Bildung von /d/, /t/, /l/, /n/). Gelegentlich fallen Kinder mit einer myofunktionellen Störung auch durch eine heisere Stimmgebung auf, die abgeklärt werden sollte.

Im klinischen Alltag kann man sich folgendes Szenario vorstellen: Auf die Frage an das Kind (4 Jahre): „Wo warst du heute?“ antwortet es: „Im Tindertarten“. (Im Kindergarten). Offensichtlich liegt hier ein Problem bei der Bildung des /k/ vor. Ein velarer Laut, das / k/, wird durch einen Alveolarlaut / $/$ ersetzt. Diesen Prozess nennt man Vorverlagerung von Velaren.

In der Untersuchung sollte nun abgeklärt werden, ob der Laut $/ \mathrm{k} /$ in irgendeiner Lautposition vom Kind schon mal gebildet wurde. Wenn es dazu keine sicheren Informationen gibt, kann dies auch durch Vor- und Nachsprechen der Laute getestet werden. Ist das Kind in der Lage, den Laut / k/ motorisch zu bilden, liegt eine phonologische Störung vor, da der Laut vom Kind nicht korrekt in seiner Lautumgebung eingesetzt werden kann. Eine phonologisch orientierte Therapie ist in diesem Fall anzuraten.

Ist das Kind nicht in der Lage, den Laut motorisch zu bilden, liegt eine phonetische Störung/Artikulationsstörung vor. Wenn der Laut phonetisch nicht korrekt produziert werden kann, kann er auch nicht korrekt in seiner Lautumgebung (phonologisch) eingesetzt werden. In so einem Fall ist vorrangig eine Artikulationstherapie zielführend. Diese kann dann, falls noch erforderlich, durch eine phonologische orientierte Therapie ergänzt werden.

\section{Artikulationsstörungen: S-Lautbildung/Sigmatismus}

Die häufigste Artikulationsstörung im Deutschen ist die Fehlbildung der S- und Zischlaute, die z. B. durch eine interdentale oder laterale Bildungsweise akustisch auffallen. Der S-Laut ist ein Konsonant, ein Frikativ (Reibelaut/ Engelaut), da die Lautbildung durch eine Enge entsteht, durch die die Luft entweicht. Es gibt 2 Möglichkeiten der S-Lautbildung im Deutschen: die apikale und die dorsale Bildungsweise.

Bei der apikalen Bildungsweise des S-Lautes bewegt sich die Zungenspitze in Richtung der oberen Schneidezähne, ohne diese zu berühren, sozusagen frei schwebend hinter den oberen Schneidezähnen (Papilla incisiva), der Phonationsstrom drängt sich durch die entstandene Öffnung ( $\mathbf{A} \mathbf{A b} \mathbf{b} . \mathbf{5}$ ). Im Rahmen der dorsalen Bildungsweise des S-Lauts liegt die Zungenspitze an der Innenkante der Unterzahnreihe. Die Vorderzunge wölbt sich zu den palatinalen Flächen der oberen Frontzähne und deren Zahndamm, wodurch eine Enge gebildet wird. Die seitlichen Zungenränder sind gehoben und liegen an den palatinalen Flächen der seitlichen oberen Zähne. Die Zungenoberfläche wird dadurch zu einer Längsrinne geformt. Der Luftstrom entweicht durch diese Rinne bis zur an den Frontzähnen gebildeten Enge ( $\mathbf{A b b} \mathbf{6} \mathbf{6}$ ).

Wenn Zahn- oder Kieferfehlstellungen oder eine myofunktionelle Störung vorliegen, kann dieses feinmotorische und sensibel hoch differenzierte System der S-Lautbildung gestört sein und es kommt zu Fehlbildungen. In diesem Falle sprechen wir von einem Sigmatismus (umgangssprachlich oft auch als „Lispeln“ bezeichnet). Es gibt verschiedene Formen des Sigmatismus, je nach Position der Zunge und wie der gebildete Luftstrom entweicht. Exemplarisch seien hier genannt:

\section{Sigmatismus interdentalis:}

Die Zungenspitze wird zwischen die geöffneten Zahnreihen vorgestreckt. Dabei entsteht ein flächiges und stumpfes Reibegeräusch, das akustisch und visuell sehr deutlich wahrzunehmen ist.

\section{Sigmatismus addentalis:}

Die Enge wird nicht wie üblich an den Alveolen gebildet, sondern an den Zähnen. Der Luftstrom entweicht dabei fächerförmig über den vorderen Zungenrücken, akustisch wie ein stumpfes, flächiges / $\mathrm{s} /$ wahrzunehmen, ähnlich wie dem englischen stimmlosen /th/.

\section{Sigmatismus lateralis:}

Bei dieser Bildungsweise entweicht der Luftstrom an den Zahnrändern, also lateral. Akustisch ähnlich einem leicht schlüpfenden Geräusch hört es sich so an, als ob mit viel Speichel gesprochen wird.

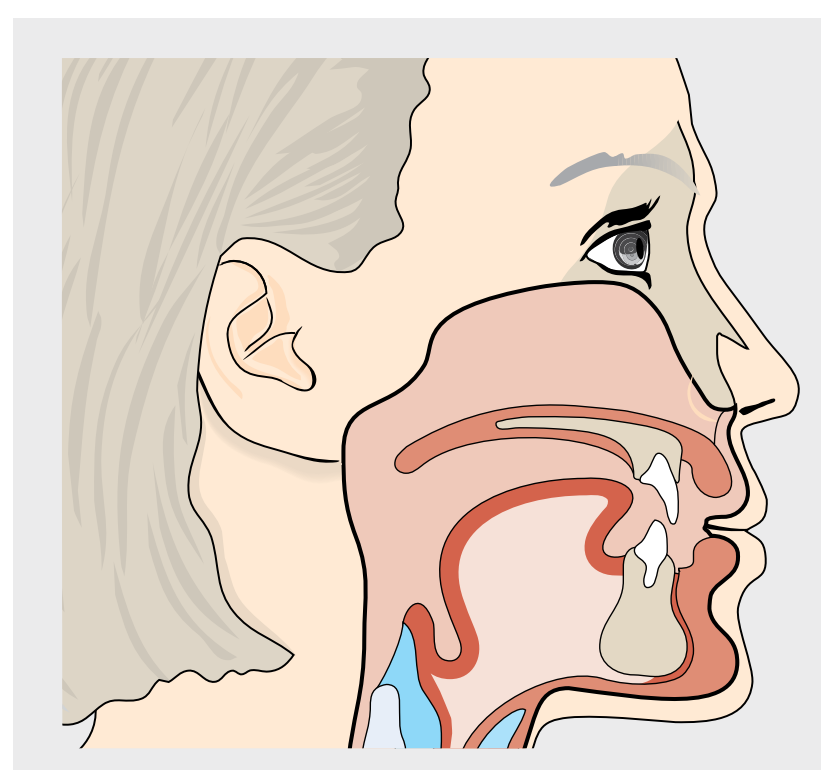

Abb. 5 Apikale Bildungsweise des S-Lauts. 


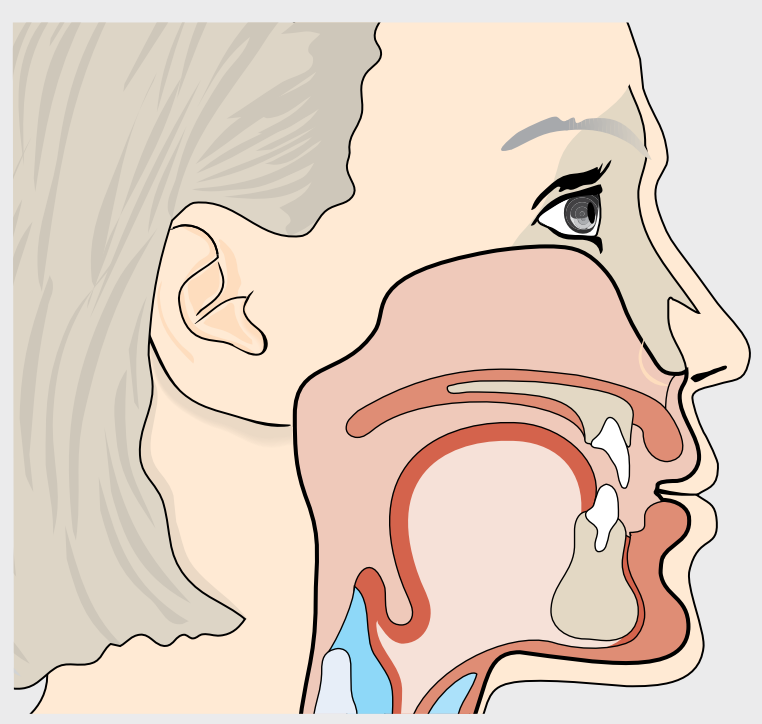

Abb. 6 Dorsale Bildungsweise des S-Lauts.

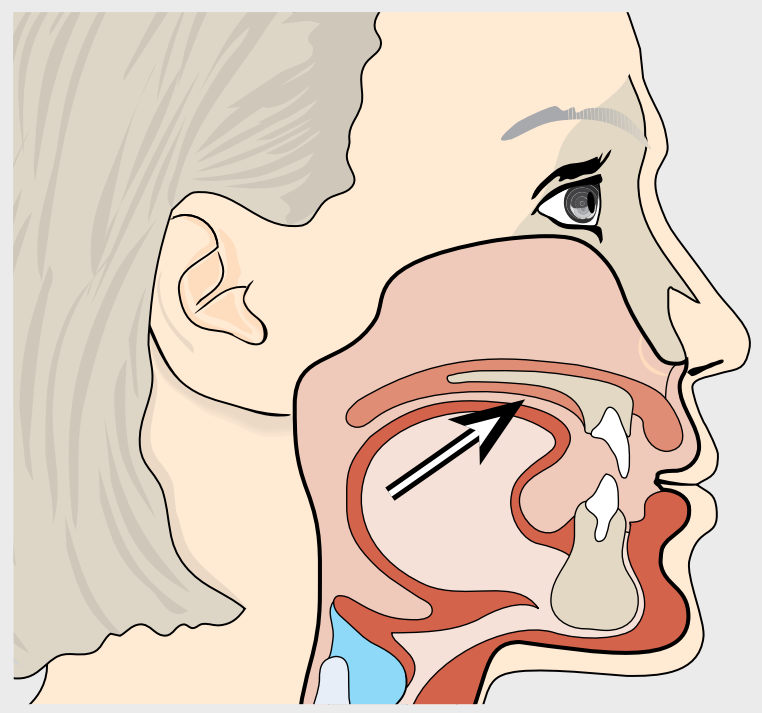

Abb. 7 Korrektes Schluckmuster.

Im Folgenden werden wichtige Symptome benannt, die einen Hinweis auf eine MFS bieten:

- viszerales/infantiles Schluckmuster,

- offene Lippenhaltung/kein optimaler Mundschluss,

- Mundatmung/behinderte Nasenatmung,

- Unterlippen verdickt und gerötet/Unterlippe nass,

- Speichel in den Mundwinkeln/Nachtsalivation,

- interdentale Zungenruhlage,

- Artikulationsstörung,

- Haltungsprobleme - oft hypotone Ganzkörperspannung.

Zu bedenken ist, dass die Ursachen hierfür sehr vielfältig sein können und nicht immer gleich und klar identifizierbar sind. Daher ist

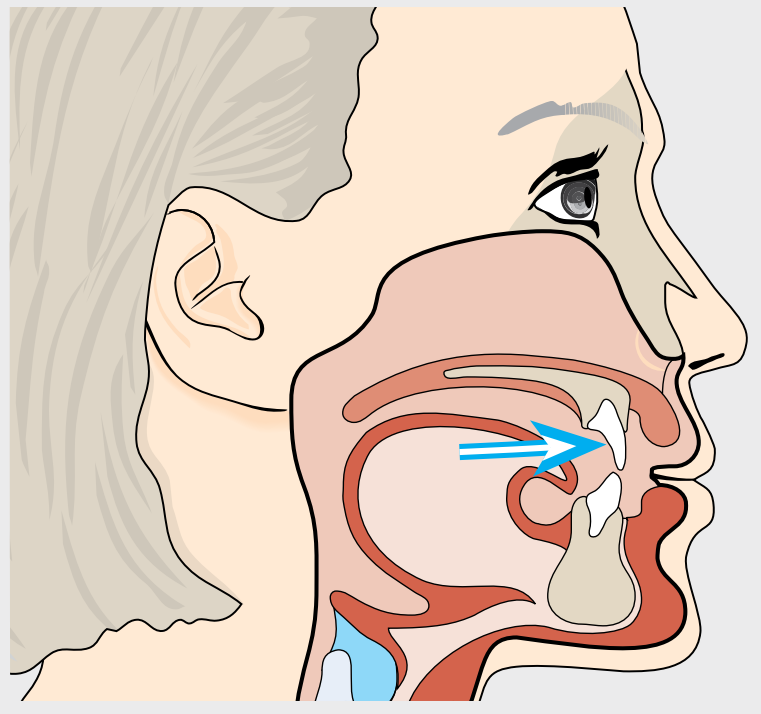

Abb. 8 Infantiles/viszerales Schluckmuster.

eine ausführlich erhobene Anamnese ein wichtiger Grundbaustein für die Diagnostik solcher Störungen. Des Weiteren ist in jedem Fall eine interdisziplinäre Diagnostik sinnvoll. Hierbei können Kinderarzt, Zahnarzt, Kieferorthopäde, Phoniater und Pädaudiologe, HNO-Arzt und Logopäden zu Rate gezogen werden. Als Ursachen für MFS können chronische Entzündungen oder Vergrößerungen der Tonsillen, häufige Infekte im Atembereich, Allergien (in Verbindung mit Behinderung der Nasenatmung), genetisch bedingte Skelettanomalien (z. B. Progenie) und Habits (z. B. Daumenlutschen) auftreten.

Der korrekte infantile/viszerale Schluckvorgang wird in den - Abb. 7 und $>$ Abb. 8 dargestellt. Als infantiles oder auch viszerales Schluckmuster wird der frühkindliche Schluckreflex bezeichnet, welcher zur Physiologie des Menschen im Kindesalter gehört [9]. Ein typisches Merkmal ist die Vorverlagerung der Zunge während des Schluckens. Da jeder Mensch unbewusst bis zu 2000-mal pro Tag den Schluckvorgang ausführt, ist es bedeutsam, dass dieser bis zum 4. Lebensjahr in ein adultes Schluckmuster umgestellt ist, bei welchem die Zunge beim Schluckakt gegen den Gaumen gedrückt wird. Der wachstumsstimulierende Effekt der Zunge führt zu einer Ausformung von Gaumen und Oberkiefer.

Anhand eines Gedankenspiels wird dies deutlich. Beim Schluckvorgang wirken Kräfte. Um die Größe der Krafteinwirkung einzuschätzen, sollte man sich Folgendes bewusst machen:

- Schluckfrequenz pro Minute: durchschnittlich 1-2 ×

- Schluckvorgang pro Tag: 1000-2000 ×

- Kraft pro Schluckvorgang: 1-2 kg

- = Krafteinwirkung pro Tag: durchschnittlich bis zu $4 \mathrm{t}$ !

Erfolgt die Kraftableitung der Zunge nicht wie physiologisch vorgesehen gegen den Gaumen, sondern gegen oder zwischen die Zähne, kann dies die Zahn- und Kieferstellung beeinflussen. So kann es zum Beispiel zur Öffnung des Bisses im Frontbereich kommen. Ebenso wären Rückfälle nach einer abgeschlossenen kieferorthopädischen Behandlung möglich, wenn das Schluckmuster nicht korrekt umgestellt wurde. 
- Tab. 3 Ausschnitt aus MFT Bogen von Steiner 1990 [10] - Beurteilung Schlucken.

\begin{tabular}{|c|c|c|}
\hline Funktion & Test & Beobachtungen \\
\hline \multirow[t]{17}{*}{ Schluckvorgang } & \multirow[t]{5}{*}{ spontan } & mit Speichel - mit fester Nahrung - mit Flüssigkeit \\
\hline & & Durchschieben der Zunge in fehlerhafte Okklusion \\
\hline & & Speichel tritt als Bläschen zwischen den Zähnen hindurch \\
\hline & & Grimasse beim Schlucken \\
\hline & & Beteiligung des M. buccinator - deutliche Lippen- und Mentaliskontraktion \\
\hline & \multirow[t]{8}{*}{ nach Aufforderung } & Mitbewegung des Kopfes - Bildung von Grübchen- Bildung von Nadelkissen \\
\hline & & mit Speichel - mit fester Nahrung - mit Flüssigkeit \\
\hline & & Durchschieben der Zunge in fehlerhafte Okklusion \\
\hline & & Speichel tritt als Bläschen zwischen den Zähnen hindurch \\
\hline & & Grimasse beim Schlucken \\
\hline & & Beteiligung des M. buccinator - deutliche Lippen- und Mentaliskontraktion \\
\hline & & Mitbewegung des Kopfes. Bildung von Grübchen. Bildung von Nadelkissen \\
\hline & & Lippen lassen sich manuell nur schwer öffnen \\
\hline & \multirow[t]{3}{*}{ PAYNE-Technik } & beim Untersuchen mit Lippenhaltern: Tendenz zum Schließen \\
\hline & & Paste zeichnet sich an Frontzähnen ab - Paste zeichnet sich an Mahlzähnen ab \\
\hline & & Horizontal gezogene Pastenstriche zeichnen sich am Gaumen ab \\
\hline & weitere Beobachtungen & \\
\hline
\end{tabular}

Bei der Abklärung einer myofunktionellen Störung ist es notwendig, folgende Parameter zu beurteilen:

- Schluckvorgang, unauffällig oder infantil/viszeral

- Mundmotorik in Ruhe und beim Schluckvorgang: Lippen, Zunge, Muskulatur, wie z. B. M. masseter, temporalis, mentalis, Kiefer, Gaumen

- Gewohnheiten, z. B. Essgewohnheiten, Präferenzen bez. der Konsistenz der Speisen

- Gesamtkörperspannung

- Atmung, z. B. Nasenatmung möglich?

- andere klinische Beobachtungen, wie z. B. chronisch entzündliche Tonsillen, Nachtsalivation, Allergien, adenoide Vegetation

Hierbei ist es sinnvoll, myofunktionelle Diagnostikbögen zu verwenden, z. B. von Jürgen Steiner ( $\triangleright$ Tab. 3, [10]) oder Anita Kittel [8].

Das viszerale/infantile Schluckmuster stellt sich normalerweise im Alter zwischen 3 und 4 Jahren auf das adulte Schluckmuster um.

\section{Ablauf einer Übungsbehandlung}

Nach einer sprachtherapeutisch erhobenen Anamnese und Befunderhebung kann ein Therapieplan erstellt werden. Die Ergebnisse und der Behandlungsplan sollten unbedingt mit dem Kind und den Bezugspersonen besprochen werden. Auch die Darstellung der unterschiedlichen Therapieschritte ist sinnvoll, um eine Verständnisabsicherung vorzunehmen.
Beim Aufbau und der Auswahl der Übungen sollte strukturiert vorgegangen werden. Wenn ein neues Schluckmuster angebahnt werden soll, wird nicht sofort mit Schluckübungen begonnen, sondern mit Übungen, die die orofaziale Dysfunktion reduzieren. Daneben sollten auch Übungen angeboten werden, die auf die bestehende Ganzkörperproblematik eingehen, z. B. auf Körperhaltung und Körpersymmetrie. Für die Muskelarbeit werden gezielte, nach Schwierigkeitsgrad gestaffelte Übungen genutzt und je nach Alter des Kindes entsprechende angeboten [8]. Dabei ist die Erfahrung des Therapeuten entscheidend. Um die Compliance und die Freude an der Therapie zu erhalten, sollten die Übungen entsprechend modifiziert werden. Hausaufgaben sind ein wichtiger Bestandteil der Behandlung. Weitere Informationen über Sprachstörungen bietet bspw. die Deutsche Gesellschaft für Sprachstörungen an [11].

\section{Ausgewählte, häufig kombiniert kieferorthopädisch-logopädische Auffälligkeiten} Im Folgenden soll ein Überblick über ausgewählte Zahnfehlstellungen [12] und in diesen Fällen häufig auftretende Sprechauffälligkeiten gewährt werden. Natürlich kann an dieser Stelle nur ein kleiner Auszug von Auffälligkeiten dokumentiert werden. Es wird ausdrücklich darauf hingewiesen, dass hier nur regelmäßig auftretende Zusammenhänge dargestellt werden, die keinesfalls bei jedem Patienten zutreffen müssen.

\section{Distalbiss}

Eine häufig auftretende Zahnfehlstellung ist eine vergrößerte sagittale Stufe ( $>$ Abb. 9). Gemeint ist damit, dass die Frontzahnstufe bei habitueller Okklusion in der Horizontalebene vergrößert ist. 


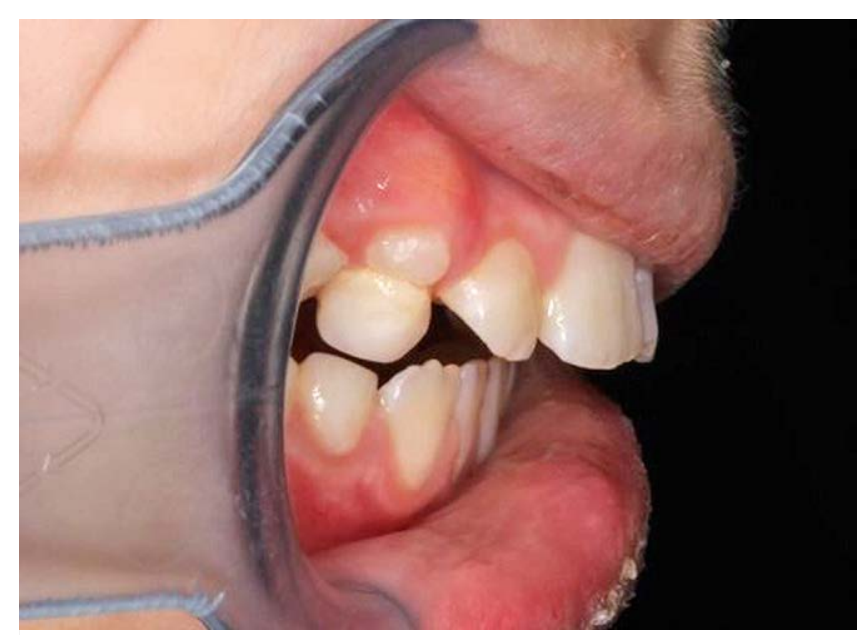

Abb. 9 Distalbiss.

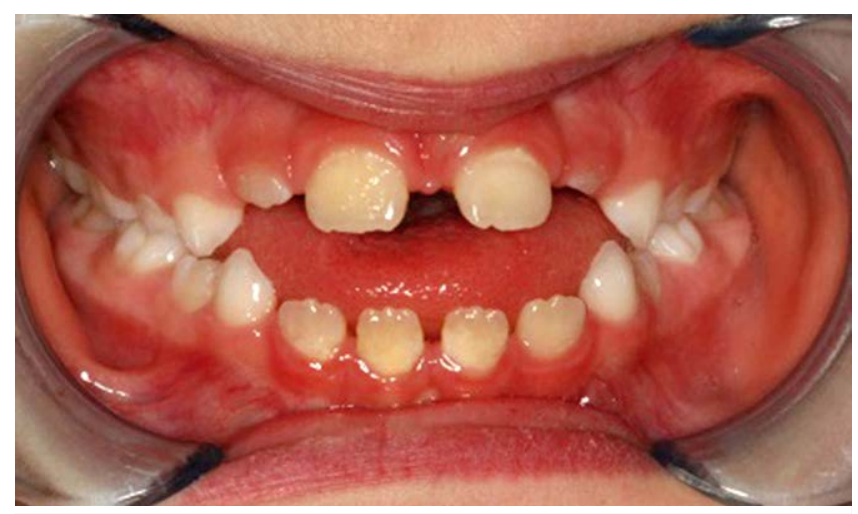

Abb. 10 Frontal offener Biss.

Die Ursachen hierfür sind vielfältig, so können sie z. B. durch eine Unterkieferrücklage oder auch Habits und Parafunktionen (Daumenlutschen, Lippeneinlagerung o. ä.) entstehen und sind kombiniert mit protrudiert stehenden oberen Inzisiven. Häufig ist der Lippenschluss (potenziell) inkompetent. Zur Übernahme der Kosten durch die Krankenkasse muss eine Stufe von über $6 \mathrm{~mm}$ messbar sein.

Bei dieser Form der Zahn- und Kieferfehlstellung sind häufige Sprechauffälligkeiten ein fehlender Lippenkontakt bei der Bildung der Labiallaute (/b/, /p/, /m/) und ein Sigmatismus addentalis. Letzteres ist dadurch gekennzeichnet, dass die Zunge bei der Lautbildung an die Unterkieferschneidezähne stößt und das /s/ an den Zähnen gebildet wird.

Im Rahmen einer kieferorthopädischen Therapie werden eine Normalisierung der Okklusionsverhältnisse und eine Reduktion der sagittalen Stufe angestrebt. Je nach Zeitpunkt der Diagnostik muss oft zunächst der Zahnbogen des Oberkiefers geweitet werden. Eine sagittale Nachentwicklung des Unterkiefers erfolgt häufig mittels funktionskieferorthopädischen Geräten, kann aber auch festsitzend therapiert werden.

Erforderlich ist aber auch eine logopädische Begleittherapie, wobei z. B. folgende Maßnahmen durchgeführt werden können.

- Übungen zur Zungenmotorik und Lippenmotorik/Verbesserung des Lippenschlusses
- Übungen zur oralen Wahrnehmung/Stereognose

- Übungen zur Verbesserung Nasenatmung

- ganzkörperliche Übungen zur Verbesserung des ganzkörperlichen Tonus

- Abgewöhnung bestehender Habits

- Korrektur Schluckmuster

Weitere Behandlungsziele sind die Abgewöhnung bestehender $\mathrm{Ha}$ bits, sowie ggf. das Trainieren eines kompetenten Lippenschlusses und das Vermeiden von Verspannungen der Kaumuskulatur.

\section{Offener Biss}

Wenn kein Kontakt von Zähnen im Front- und/oder Seitenzahngebiet bei habitueller Okklusion besteht, spricht man von einem offenen Biss ( $\triangleright$ Abb. 10). Beachtung finden dabei vollständig durchgebrochene Zähne. Bis zum 18. Lebensjahr wird eine Behandlung ab über 2 mm Öffnung des Bisses durch die Krankenkasse übernommen.

Eine Form des offenen Bisses ist der dentoalveolär-offene Biss. Dabei ist das Wachstumsmuster des Patienten neutral, die Ätiologie liegt meist in der fehlenden Umstellung des infantilen Schluckmusters, Zungenpressen bzw. exzessivem und langem Gebrauch von Schnullern oder Fingerlutschen.

Wenn aber auch das Wachstumsmuster des Patienten von der Norm abweicht und sich als vertikales Wachstumsmuster darstellt, spricht man von einem skelettaloffenen Biss. Dieser ist vorwiegend erblich bedingt und meist durch eine vergrößerte Untergesichtshöhe charakterisiert. Seltener können offene Bisse auch durch Erkrankungen, z. B. Rachitis oder iatrogen, bedingt sein.

Die Priorität bei einem dentoalveolär-offenen Biss liegt in der Ursachenbeseitigung. Wichtiger Aspekt ist die Umstellung des infantilen Schluckmusters. Durch ein bestehendes infantiles Schluckmuster wird die Zunge tausendfach am Tag zwischen die Zahnreihen der Front gepresst, sodass der offene Biss häufig persistiert. Die Umstellung des infantilen Schluckmusters sowie evtl. vorhandene Habits müssen abtrainiert werden, um einem Rezidiv vorzubeugen.

Häufig auftretende artikulatorische Auffälligkeiten in Kombination mit einem offenen Biss sind der Sigmatismus interdentalis bzw. eine interdentale Bildungsweise des/sch/ und/oder $/ \mathrm{ch}^{1} /$.

Oft sehen wir bei dieser Fehlstellung auch eine interdentale Bildungsweise der Laute /I/, /d/, /t/ und /n/. Auch ein Sigmatismus lateralis kann akustisch wahrzunehmen sein oder eine laterale Bildungsweise des /sch/. Die nicht normgerechte Aussprache des / sch/ wird als Schetismus bezeichnet.

Im Rahmen des skelettal-offenen Bisses kann es aufgrund von ungünstigen Wachstumsschüben erforderlich sein, einen Schluss des Bisses durch ein kombiniert kieferorthopädisch-kieferchirurgisches Vorgehen durchzuführen.

Im Rahmen einer kieferorthopädischen Therapie wird ein Schluss des offenen Bisses angestrebt. Häufig findet man bei Abstellen des infantilen Schluckens bereits eine Besserung der Bisssituation. Anschließend kann herausnehmbar oder festsitzend im Ober- und Unterkiefer behandelt werden.

Folgende Inhalte könnten zu Beginn der logopädischen Behandlung z. B. zielführend sein:

- Abgewöhnung Habits,

- Regulierung der Zungen, Wangen- und Lippenmuskulatur, 


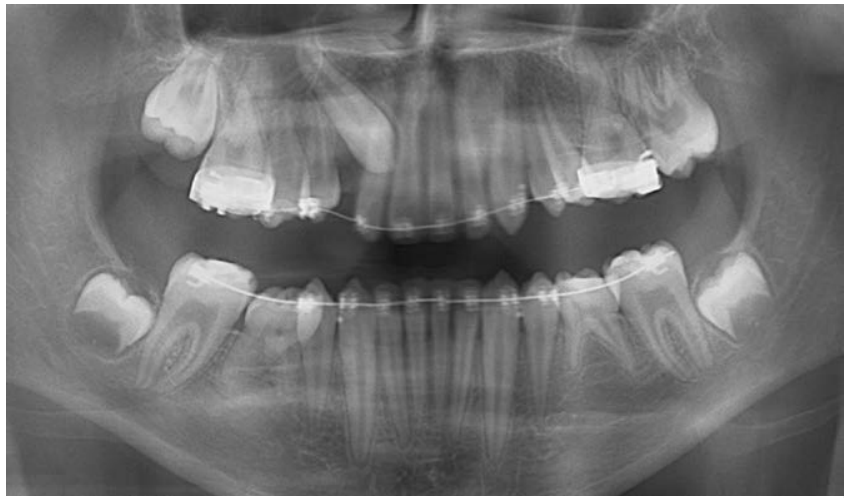

Abb. 11 Verlagerter Zahn 13; 35 und 45 nicht angelegt.

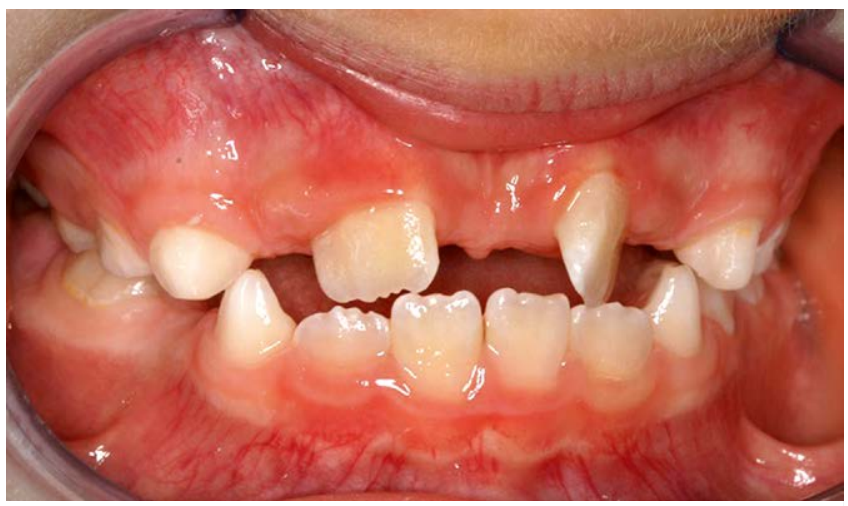

Abb. 12 Zustand nach Entfernung eines Mesiodens.

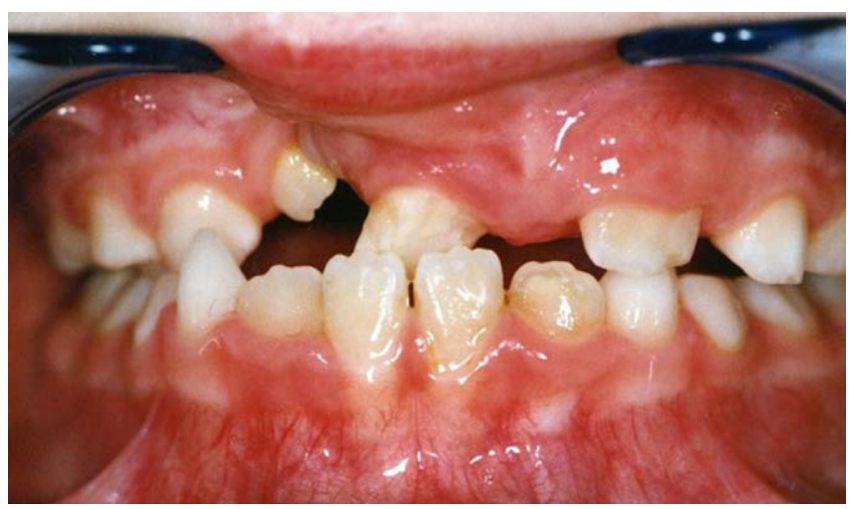

Abb. 13 Patient mit Lippen-, Kiefer- und Gaumenspalte, Zustand nach Gaumen- und Lippenspaltverschluss.

- ganzkörperliche Übungen zur Verbesserung der Körperstatik/ zur Regulierung der Körperspannung.

\section{Durchbruchsstörungen}

Unter Durchbruchsstörungen versteht man Veränderungen, weshalb ein Zahn nicht normgerecht durchbrechen kann. Hierunter fallen auch retinierte oder verlagerte Zähne ( $\mathbf{A}$ Abb. 11). Ursache einer Durchbruchsstörung können vielfältig sein, so z. B. persistierende Milchzähne, vorzeitiger Milchzahnverlust mit Aufwanderung des Seitenzahngebietes und resultierendem Platzmangel, Trauma- ta, Hyperdontien (z. B. Mesiodens, \ Abb. 12) oder Neubildungen im Kieferbereich.

Natürlich kann v. a. bei geschlossener Zahnreihe eine Durchbruchsstörung ohne jegliche Sprechauffälligkeit auftreten. Häufig kommt ein Sigmatismus stridens vor, v. a. bei Diastema mediale. Auch ein Sigmatismus interdentalis ist möglich. Im Seitenzahngebiet ist meist ein Sigmatismus oder Schetismus lateralis auffällig.

In jedem Fall sollte versucht werden, das Durchbruchshindernis zu eruieren und zu entfernen. Als nächster Schritt muss geprüft werden, ob eine Einstellung der retinierten/verlagerten Zahnes kieferorthopädisch möglich ist. Falls notwendig, wird dieser Zahn chirurgisch freigelegt und anschließend mit einer kieferorthopädischen Apparatur eingestellt. Aus sprachtherapeutischer Sicht könnten hier folgende Inhalte angeboten werden:

- mundmotorische Übungen zur Verbesserung der Myofunktion

- Korrektur der Lautbildung/Artikulationstherapie

\section{Kraniofaziale Anomalien}

Unter kraniofazialen Anomalien versteht man anlagebedingte oder erworbene Fehlbildungen, die einer interdisziplinären Behandlung bedürfen. Neben Lippen-, Kiefer- und Gaumenspaltbildungen ( $\triangleright$ Abb. 13) zählen zu dieser Gruppe auch Syndrome wie z. B. das Goldenhar- Syndrom oder Morbus Crouzon und einige mehr.

Die Rehabilitation dieser Patienten ist häufig sehr komplex. Jede Anomalie stellt eine Herausforderung für die Behandelnden und den Patienten dar und bedarf eines umfassenden therapeutischen Behandlungskonzeptes.

Bei der Betrachtung von Lippen-, Kiefer- und Gaumenspalten wird meist eine maxilläre Hypoplasie deutlich. Durch den Narbenzug im Oberkiefer und nicht selten auftretende Nichtanlagen der oberen seitlichen Schneidezähne ist das Wachstum im Oberkiefer meist eingeschränkt möglich. Eine resultierende progene Verzahnung kommt beständig vor. Die Behandlung von Patienten mit Lippen-, Kiefer-, Gaumenspalten beginnt häufig schon in der 1. Phase des Wechselgebisses mit dem Versuch der transversalen Weitung und sagittalen Nachentwicklung des Oberkiefers sowie der Überstellung der Frontzähne.

Beim Vorliegen einer Lippen-, Kiefer-, Gaumenspalte besteht häufig auch eine Rhinophonie oder Rhinolalie (umgangssprachlich auch unter Näseln bekannt). Rhinophonie meint eine pathologische Veränderung des Stimmklangs, bedingt durch zu viel oder zu wenig Luft, die bei der Stimmbildung/beim Sprechen über die Nase austritt. Dadurch zeigt sich eine akustisch auffällige Stimmgebung. Treten durch diese Einschränkungen auch Lautfehlbildungen auf, wird dies als Rhinolalie bezeichnet.

Die klanglichen Veränderungen und Lautfehlbildungen können vereinzelt, aber auch zusammen auftreten. Je nach dem, was stärker imponiert, spricht man dann eher von einer Rhinophonie, wenn hauptsächlich der Stimmklang betroffen ist, oder von einer Rhinolalie, wenn die Lautbildung stärker betroffen ist.

Es wird unterschieden in:

- Rhinophonia aperta/Rhinolalia aperta/Hyperrhinophonie (offenes Näseln), hervorgerufen durch ein Zuviel an Nasenresonanz. Es liegt ein mangelhafter Verschluss der Nasenräume während der Stimmgebung und/oder Sprechens vor, die Luft entweicht dabei in erheblicher Menge durch die Nase, anstatt durch den Mund. 
- Rhinophonia clausa/Rhinolalia clausa/Hyporhinophonie (geschlossenes Näseln), hervorgerufen durch ein Zuwenig an Nasenresonanz. Die Luft entweicht nicht durch die Nase sondern nur durch den Mund, akustisch oft durch einen dumpfen, verschnupften Klang zu identifizieren.

- Rhinophonia mixta/Rhinolalia mixta (gemischtes Näseln), hervorgerufen durch einen fehlenden oder mangelhaften Verschluss der Nasenräume und einer gleichzeitig vorliegenden Einschränkung der Nasenresonanz.

Es sei nochmals erwähnt, dass bei kraniofazialen Anomalien eine Abklärung der Hörstörung unbedingt anzuraten ist.

Eine Rhinophonie/Rhinolalia kann organisch oder funktionell bedingt sein. Bei einem offenen Näseln können z. B. als organische Ursache angeborene Lippen-, Kieferund Gaumen-Spaltformen, Narben nach Operationen, zentrale Lähmungen des Velums bei Erkrankungen des Gehirns infrage kommen. Funktionelle Ursachen können bspw. eine falsche Sprechgewohnheit und habituelles Herabhängenlassen des Gaumensegels z. B. nach Tonsillektomie sein. Beim geschlossenen Näseln sind typische organische Ursachen raumverengende Faktoren wie z. B. Mandelhyperplasie oder allergische Reaktionen, weiterhin Nasenfehlbildungen und neurologische Erkrankungen. Bei den funktionellen Ursachen kommen oft habituelle Faktoren infrage, die zu einer Dauerkontraktion des Gaumensegels führen.

Betrachtet man die Artikulation, so sind meist die nasalen Laute (/m/, /n/, /ng/) oder velare Laute auffällig. Bei Vorliegen einer Lippenspalte ist der Lippenkontakt der Labiallaute /b/,/p/, /m/ und der Vokale wie /o/, /u/ oft nur eingeschränkt oder nicht möglich, ein Sigmatismus addentalis ist bei progener Verzahnung häufig. Ein Schetismus ergänzt nicht selten dieses Bild.

Logopädische/sprachtherapeutische Intervention ist hier unbedingt anzuraten. Inhalte der Therapie könnten z. B. sein:

- Übungen zur Stärkung und Aktivierung der Myofunktion

- ganzkörperliche Übungen zur Verbesserung der Körperstatik/ zur Regulierung der Körperspannung

- Saug- und Pusteübungen zur Aktivierung der velaren Muskulatur

- im Rahmen des Möglichen, Korrektur der Artikulation

- Verbesserung der Stimmfunktion

Im Therapiekonzept der Rehabilitation von Spaltträgern ist es notwendig, dass die interdisziplinäre Zusammenarbeit gewährleistet ist. Neben der Anfertigung von Mund-, Nasentrennplatten, der kieferorthopädischen Behandlung, multiplen operativen Eingriffen ist die phoniatrisch- pädaudiologische, ohrenärztliche und logopädische Untersuchung und Behandlung essenziell.

\section{Zusammenfassung - worauf Sie als Zahnarzt achten sollten}

Im Rahmen des Zahnwechsels sollte ein Zahnarzt regelmäßig auch die Zahn- und Kieferstellung kontrollieren. Abweichungen vom eugnathen Gebiss sollten notiert und bei auffälligen Unregelmäßigkeiten und progredienten Veränderungen dem Kieferorthopäden vorgestellt werden. Grundsätzlich sollten dabei z. B. auf Auffälligkeiten der sagittalen Stufe, des Overbites, Unregelmäßigkeiten des
Zahndurchbruches und transversale Abweichungen geachtet werden. Es ist von enormer Wichtigkeit, dass falls erforderlich eine gute Kommunikation zwischen Zahnarzt und Kieferorthopäden stattfindet. Bei komplexen Behandlungen sind Absprachen bezüglich späterer konservierender Aufbauten, prothetischer Versorgungen und Implantationen dringend notwendig, um ein stabiles Behandlungsergebnis zu erreichen.

Zudem können eine Überprüfung der Sprachbildung unter Kenntnis der kindlichen Sprachentwicklung und die Beurteilung einer myofunktionellen Störung durchgeführt werden. Wichtige Anhaltspunkte bieten hierbei die Lautbildung, der Schluckvorgang, die Atmung, aber auch Habits. Eine sorgfältige Anamnese bildet hier die Grundlage.

Falls Normabweichungen auffallend sind, sollte eine Überweisung zur entsprechenden Fachgruppe erfolgen. Der Fachzahnarzt für Kieferorthopädie wird entscheiden, ob eine Behandlung notwendig ist und erforderlichenfalls im Folgenden eine Behandlung zur Behebung der Fehlstellung vornehmen.

Seit dem 01.07.2017 ist die Heilmittelverordnung durch Zahnärzte erstmals in eigener Richtlinie geregelt (Download z. B. unter www.kzbv.de). Laut Heilmittelkatalog ist eine Sprech- und Sprachtherapie bei Störungen des Sprechens (SPZ, z. B. durch Zahn- und Kieferfehlstellungen), Störungen des oralen Schluckakts (SCZ, z. B. bei viszeralem Schluckmuster) und orofazialen Funktionsstörungen (OFZ, z. B. bei angeborenen kranio- und orofazialen Fehlbildungen) möglich. Die Erstverordnung darf über 10 Sitzungen ausgeschrieben werden, wobei diese 1-3-mal wöchentlich durchzuführen sind. Die Sprechund Sprachtherapie erfolgt meist über 30 oder 45 Min. Für eine Folgeverordnung sind nochmals zehn Sitzungen verschreibbar. In der Regel sollten nicht mehr als 30 Einheiten notwendig sein [13].

\section{FAZIT}

- Bei jedem Kind sollte eine regelmäßige Kontrolle der Gebissentwicklung in den Phasen des Milch- und Wechselgebisses sowie ggf. der rechtzeitige Beginn einer erforderlichen kieferorthopädischen Therapie erfolgen.

- Bei Auffälligkeiten der Sprachentwicklung oder Lautbildung ist eine Untersuchung und ggf. Therapie durch Phoniater und Pädaudiologe, HNO-Arzt und/oder Logopäden ratsam.

- Im Rahmen der Heilmittelverordnung (2017) ist eine Sprech- und Sprachtherapie durch den Zahnarzt bei Störungen des Sprechens, Schluckens oder bei orofazialen Funktionsstörungen möglich.

- Je nach Schweregrad der Anomalie ist ein interdisziplinäres Behandlungskonzept notwendig, um ein optimales Behandlungsergebnis zu erreichen und zu stabilisieren.

Interessenkonflikt

Die Autoren geben an, dass kein Interessenkonflikt besteht. 


\section{Erstveröffentlichung}

Dieser Beitrag wurde erstveröffentlicht in: Zahnmedizin up2date 2017; 11 (5): 511-527.

\section{Literatur}

[1] Klink-Heckmann U, Bredy E. Orthopädische Stomatologie. 2. Aufl. Leipzig: Johann Ambrosius Barth Verlag; 1980

[2] Hägg U, Taranger ]. Dental emergence stages and the pubertal growth spurt. Acta Odontol Scand 1981; 39: 295-306

[3] Leighton BC. Variationen der normalen Gebissentwicklung von der Geburt bis zum Erwachsenenalter. Fortschr Kieferorthop 1978; 39: 181-195

[4] Harzer W. Lehrbuch der Kieferorthopädie. 1. Aufl. München, Wien: Carl Hanser Verlag; 1999

[5] Harzer W. Kieferorthopädie (Checklisten Zahnmedizin). Stuttgart: Thieme; 2011

[6] Richtlinien des Bundesausschusses zur kieferorthopädischen Behandlung. Im Internet: http://www.kzbv.de/kieferorthopaedie-2004.download.7d16079a6c85714d65b925127b4e1c89.pdf; Stand: 05.07.2017
[7] Fox-Boyer A. Kindliche Aussprachestörungen Phonologischer Erwerb-Differentialdiagnostik-Therapie. 6. Aufl. Idstein: Schulz-Kirchner Verlag; 2003

[8] Kittel A. Myofunktionelle Therapie. 3. Aufl. Idstein: Schulz-Kirchner Verlag; 1998

[9] Wirth G. Sprachstörungen - Sprechstörungen - kindliche Hörstörungen: Lehrbuch für Ärzte, Logopäden und Sprachheilpädagogen. 5. Aufl. Köln: Deutscher Ärzte-Verlag; 2000

[10] Steiner J. MFT Diagnostikbogen Befunderhebung bei Mundmuskelfunktionsstörungen. Leverkusen: Steiner Verlag; 1990

[11] Deutsche Gesellschaft für Sprachheilpädagogik. Informationshefte über Sprachstörungen. Im Internet: https://www.dgs-ev.de/index. php?id = 74 Stand 05.07.2017

[12] Sander FM, Schwenzer N, Ehrenfeld M. Zahn-Mund- und Kieferheilkunde. Kieferorthopädie. 2. Aufl. Stuttgart: Thieme Verlag; 2011

[13] Katalog verordnungsfähiger Heilmittel - Heilmittelkatalog ZÄ. Im Internet: https://www.g-ba.de/downloads/17-98-4291/2016-12-15 HeilM-RL-ZAE_Erstfassung_Heilmittelkatalog.pdf. Stand: 19.09.2017 